Mathematical Proceedings of the Cambridge Philosophical Society

http://journals.cambridge.org/PSP

Additional services for Mathematical Proceedings of the Cambridge

Philosophical Society:

Email alerts: $\underline{\text { Click here }}$

Subscriptions: $\underline{\text { Click here }}$

Commercial reprints: $\underline{\text { Click here }}$

Terms of use : $\underline{\text { Click here }}$

\title{
On semiconjugation of entire functions
}

WALTER BERGWEILER and A. HINKKANEN

Mathematical Proceedings of the Cambridge Philosophical Society / Volume 126 / Issue 03 / May 1999, pp 565 - 574

DOI: null, Published online: 08 September 2000

Link to this article: http://journals.cambridge.org/abstract_S0305004198003387

How to cite this article:

WALTER BERGWEILER and A. HINKKANEN (1999). On semiconjugation of entire functions. Mathematical Proceedings of the Cambridge Philosophical Society, 126, pp 565-574

Request Permissions : $\underline{\text { Click here }}$ 


\title{
On semiconjugation of entire functions
}

\author{
By WALTER BERGWEILER \\ Mathematisches Seminar, Christian-Albrechts-Universität zu Kiel, \\ Ludewig-Meyn-Str. 4, D-24098 Kiel, Germany \\ e-mail: bergweiler@math.uni-kiel.de \\ AND A. HINKKANEN $\dagger$ \\ Department of Mathematics, University of Illinois at Urbana-Champaign, Urbana, \\ Illinois 61801, USA \\ e-mail: aimo@math.uiuc.edu
}

(Received 8 December 1997; revised 3 April 1998)

\section{Abstract}

Let $f$ and $h$ be transcendental entire functions and let $g$ be a continuous and open map of the complex plane into itself with $g \circ f=h \circ g$. We show that if $f$ satisfies a certain condition, which holds, in particular, if $f$ has no wandering domains, then $g^{-1}(J(h))=J(f)$. Here $J(\cdot)$ denotes the Julia set of a function. We conclude that if $f$ has no wandering domains, then $h$ has no wandering domains. Further, we show that for given transcendental entire functions $f$ and $h$, there are only countably many entire functions $g$ such that $g \circ f=h \circ g$.

\section{Introduction and results}

The Fatou set $F(f)$ of an entire or rational function $f$ is the set where the family $\left\{f^{n}\right\}$ of iterates of $f$ is normal and the Julia set $J(f)$ is its complement. These sets play a fundamental role in complex dynamics (see $[\mathbf{5}, \mathbf{1 0}, \mathbf{2 1}, \mathbf{2 3}]$ for an introduction to this theory).

Now let $f$ and $h$ be entire functions and let $g: \mathbb{C} \rightarrow \mathbb{C}$ be a non-constant continuous function such that

$$
g \circ f=h \circ g
$$

Then we say that $f$ and $h$ are semiconjugated (by $g$ ) and call $g$ a semiconjugacy.

Theorem 1. If $f$ and $h$ are transcendental entire functions, if $g$ is a non-constant continuous function and if (1) holds, then

$$
g(J(f)) \subset J(h) .
$$

If, in addition, $g(\mathbb{C})$ is an open set and, in particular, if $g$ is an open mapping, then $\mathbb{C} \backslash g(\mathbb{C})$ contains at most one point.

$\dagger$ A.H. was partially supported by the U.S. National Science Foundation grant DMS 94-00999. 
The special case when $g$ is entire is important and Theorem 1 is easy to prove in this case. Theorem 1 is also easy to prove if we assume that $g$ is open or discrete.

Even in the case when $g$ is entire, however, it is not clear whether we also have

$$
g^{-1}(J(h)) \subset J(f)
$$

and thus

$$
g^{-1}(J(h))=J(f) .
$$

If $g$ is a homeomorphism of $\mathbb{C}$ onto itself satisfying (1), then $g$ is called a conjugacy. In this case (4) clearly holds. It is also known that (4) holds if $g(z)=e^{z}$ (see [8]). Here it suffices to assume that $f$ is a holomorphic self-map of $\mathbb{C} \backslash\{0\}$. In [9] it was shown that (4) holds if $g$ is entire and if there exists an entire function $k$ such that $f=k \circ g$ and $h=g \circ k$. Note that if $f$ and $h$ have this special form, then (1) is always satisfied.

In order to state a further condition implying (4) we recall that a component $U$ of $F(f)$ is called a wandering domain if $f^{m}(U) \cap f^{n}(U)=\varnothing$ for $m \neq n$. A famous theorem of Sullivan [24] says that rational functions do not have wandering domains. Transcendental entire functions, however, may have wandering domains, but certain classes of functions with no wandering domains are known (see [6, sections 4.5, 4.6] for further discussion and references).

A further concept that we need is the set $A(f)$ where the iterates of a transcendental entire function $f$ tend to $\infty$ about as fast as possible. Given such an $f$, we shall see in Section 4 that

$$
\lim _{n \rightarrow \infty} \frac{\log \log M\left(R, f^{n}\right)}{n}=\infty
$$

for all large $R>0$ and that

$$
A(f)=\left\{z \in \mathbb{C}: \text { there exists } L \in \mathbb{N} \text { such that }\left|f^{n}(z)\right|>M\left(R, f^{n-L}\right) \text { for } n>L\right\}
$$

is not empty for such $R$. For a further discussion of the set $A(f)$ we refer to Section 4, but note that if $f$ does not have wandering domains then $A(f) \subset J(f)$; see Lemma 4 below.

Theorem 2. Let $f$ and $h$ be transcendental entire functions and let $g: \mathbb{C} \rightarrow \mathbb{C}$ be open and continuous such that $g \circ f=h \circ g$. If $A(f) \subset J(f)$ then $g^{-1}(J(h))=J(f)$. In particular, this is the case if $f$ does not have wandering domains.

Consider the special case when $f=h$ and $g$ is entire. We then have $f \circ g=g \circ f$ and say that $f$ and $g$ commute. Theorem 2 implies that $g^{-1}(J(f))=J(f)$ which means that $J(f)$ is completely invariant under $g$. Now $J(g)$ is known to be the smallest closed completely invariant set with at least three points (see, for example [5, p. 67] for the special case of rational functions). We deduce that $J(g) \subset J(f)$. We thus have the following result.

Corollary. Let $f$ and $g$ be commuting transcendental entire functions. If $f$ does not have wandering domains or, more generally, if $A(f) \subset J(f)$ then $J(g) \subset J(f)$.

The conclusion that $J(g) \subset J(f)$ if $f$ does not have wandering domains was obtained by Langley [20] under an additional growth restriction on $g$.

Of course the corollary implies that if neither $f$ nor $g$ has wandering domains, then 
$J(f)=J(g)$. It is conjectured that this remains valid in general, i.e. even if $f$ and $g$ are allowed to have wandering domains. It is known to be true for rational functions (see [14, pp. 364-365], [19, p. 143] or [3, section 4]).

There are other results for commuting entire functions which have a generalization to the situation of a semiconjugacy. One such result is:

Theorem 3. Let $f$ and $h$ be entire functions such that $f$ is either transcendental or a polynomial of degree at least 2 and $h$ is not the identity mapping. Then there are only countably many entire functions $g$ such that (1) holds.

Of course, if $h$ is the identity mapping then (1) reads $g \circ f=g$, which is satisfied by all constant maps $g$ but not by any non-constant map $g$ unless $f(z)=\omega z+c$ for some root of unity $\omega$ and some $c \in \mathbb{C}$ (see, for example [17]). If $f$ has this form, then there are uncountably many non-constant solutions $g$ of the equation $g \circ f=g$.

Theorem 3 generalizes the result of Baker in [1, theorem 1, p. 244] where it was proved that if $f$ is a given entire function, either transcendental or a polynomial of degree at least two, then there are only countably many entire functions $g$ commuting with $f$.

Theorem 4. Let $f$ and $h$ be transcendental entire functions and let $g: \mathbb{C} \rightarrow \mathbb{C}$ be open and continuous such that (1) holds. If $f$ has no wandering domains then $h$ has no wandering domains.

Theorem 4 generalizes the result in [9] where the conclusion was obtained if $g$ is entire and if $f=k \circ g$ and $h=g \circ k$ for some entire function $k$. This was used in [9] to exhibit certain new classes of entire functions with no wandering domains. If $f=k \circ g$ and $h=g \circ k$ as in [9], then, by symmetry, $f$ has wandering domains if and only if $h$ has wandering domains. In the situation of Theorem 4 , however, it is possible that $f$ has wandering domains while $h$ does not. An example is $f(z)=z+e^{z}+1+2 \pi i$, $g(z)=e^{z}$ and $h(z)=z e^{z+1}$.

\section{An example}

We give an example which shows that the non-constant continuous function $g$ need not be open or discrete in order to satisfy (1), even if $f=h$ so that $f$ and $g$ commute. The same example shows that for a given transcendental entire $f$ there can sometimes be uncountably many non-constant, continuous, and non-entire functions $g$ commuting with $f$ (then also (1) holds with $f=h$ ).

First choose a number $R \in(0,1)$ and then a continuous function $a:\left[R^{2}, R\right] \rightarrow(0,1)$ such that $a\left(R^{2}\right)=a(R)^{2}$. Note that then $a$ is non-constant. The condition $a\left(r^{2}\right)=a(r)^{2}$ extends the definition of $a$ uniquely to $r \in(0,1)$. Define also $a(0)=0$ and $a(r)=r$ for all $r \in[1,+\infty)$. Then $a:[0,+\infty) \rightarrow[0,+\infty)$ is continuous and satisfies $a\left(r^{2}\right)=a(r)^{2}$ for all $r \geqslant 0$.

Next define $G: \mathbb{C} \rightarrow \mathbb{C}$ by $G\left(r e^{i \theta}\right)=a(r) e^{i \theta}$. Then $G\left(z^{2}\right)=G(z)^{2}$ for all $z \in \mathbb{C}$. Clearly $a$ can be chosen so that $G$ is neither discrete nor open. Now (1) holds with $f(z)=h(z)=z^{2}$ and $g=G$.

To get an example with transcendental functions $f$ and $h$, suppose that $f=h$ is transcendental, that $f$ has a superattracting fixed point $z_{0}$ whose immediate basin of attraction $A$ is bounded and bounded by a Jordan curve, and whose basin of 
attraction contains no singularity of $f^{-1}$ other than $z_{0}$, such that $f(z)-z_{0}$ has a zero of order 2 at $z_{0}$. Let $\varphi$ be a conformal map of $A$ onto the unit disk $D$ with $\varphi\left(z_{0}\right)=0$, and note that $\varphi$ conjugates $f$ to $z^{2}$.

For $z \in A$ define $g(z)=\varphi^{-1}(G(\varphi(z)))$. Then $g(f(z))=\varphi^{-1}(G(\varphi(f(z))))=$ $\varphi^{-1}\left(G\left(\varphi(z)^{2}\right)\right)=\varphi^{-1}\left(G(\varphi(z))^{2}\right)=f\left(\varphi^{-1}(G(\varphi(z)))\right)=f(g(z))$ for $z \in A$.

If $B$ is a component of the basin of attraction of $z_{0}$ with $f^{n}(B)=A$ where $n$ is minimal, define $g(z)=f^{-n}\left(g\left(f^{n}(z)\right)\right) \in B$ for all $z \in B$, with the appropriate branch of $f^{-n}$.

Since $G$ coincides with the identity mapping on $\partial D$ and since $\varphi$ extends continuously and bijectively to $\partial A$, we see that $g$ extends continuously to $\partial A$ and $\partial B$ by setting $g(z)=z$ there. Finally, we set $g(z)=z$ when $z$ is outside the closure of the basin of attraction of $z_{0}$. Then $g$ is a continuous non-constant function commuting with $f$, hence satisfying (1) with $h=f$.

Obviously $g$ need not be discrete or open and there are uncountably many possible choices for $g$.

We still need to find a transcendental entire function $f$ with the required properties. The function $f(z)=c\left(e^{z^{2}}-1\right)$ satisfies all the conditions provided that $c$ is a sufficiently large positive number. Note that for any non-zero $c, z_{0}=0$ is a superattracting fixed point of $f$ and that $f$ has a zero or order 2 at the origin. The set of singularities of $f^{-1}$ is $\{0,-c\}$. If $c$ is large enough, we have $|f(z)|>1$ whenever $|z|=1$. This implies that the component $W$ of $g^{-1}(D)$ that contains 0 satisfies $\bar{W} \subset D$ and that the immediate basin of attraction $A$ of 0 satisfies $\bar{A} \subset W$. Moreover, $f$ is a proper map of degree 2 from $W$ onto $D$. Thus, in the terminology of $[\mathbf{1 0}$, section VI-1] or [12], the triple $(f ; W, D)$ is a polynomial-like mapping of degree 2 . The basic result about polynomial-like mappings ([10, theorem VI-1.1] or [12, theorem 1]) says that there exist a quasiconformal mapping $\psi$ and a polynomial $p$ of degree 2 such that $f(z)=\psi\left(p\left(\psi^{-1}(z)\right)\right)$ for $z \in W$. Since 0 is a superattracting fixed point of $f$ we deduce that $p$ has a superattracting fixed point and by suitably normalizing $\psi$ we may assume that $p(z)=z^{2}$. This implies that $A=\psi(D)$ and $\partial A=\psi(\partial D)$. Thus $\partial A$ is not only a Jordan curve but a quasicircle, and $f$ is conjugate to $z \mapsto z^{2}$ in $A$. Similar reasoning can be found in the proof of theorem 2 in [7, p. 529].

If $c$ is large enough, we also have $f^{n}(-c) \rightarrow \infty$ as $n \rightarrow \infty$. Thus $z_{0}=0$ is then the only singularity of $f^{-1}$ in the basin of attraction of $z_{0}=0$. This completes the construction of the example.

\section{Proof of Theorem 1}

Let the assumptions of Theorem 1 be satisfied. Recall that, by Baker's result [2], $J(f)$ is the closure of the repelling periodic points of $f$. Suppose that $b \in J(f)$. Then $b=\lim _{n \rightarrow \infty} z_{n}$ where $f^{k_{n}}\left(z_{n}\right)=z_{n}$, say, and all the $z_{n}$ are distinct repelling periodic points of $f$, and $k_{n}$ tends to infinity. Furthermore, $g(b)=\lim _{n \rightarrow \infty} g\left(z_{n}\right)$ and by (1), it is seen that $h^{k_{n}}\left(g\left(z_{n}\right)\right)=g\left(z_{n}\right)$ for all $n$. We shall show that $g\left(z_{n}\right) \in J(h)$ for all $n$. It then follows that $g(b)=\lim _{n \rightarrow \infty} g\left(z_{n}\right) \in J(h)$ and thus (2) holds.

To show that $g\left(z_{n}\right) \in J(h)$, suppose that $g\left(z_{n}\right) \in N(h)$. Since $h^{k_{n}}\left(g\left(z_{n}\right)\right)=g\left(z_{n}\right)$, the point $g\left(z_{n}\right)$ is then an attracting, superattracting or Siegel fixed point of $h^{k_{n}}$. Now $g(\mathbb{C})$ is a connected set containing more than one point. Hence we may choose a small neighbourhood $V_{n}$ of $g\left(z_{n}\right)$ so that the set $A=g(\mathbb{C}) \backslash \bigcup_{p=0}^{\infty} h^{p k_{n}}\left(V_{n}\right)$ contains at least two points. This implies that $B=g^{-1}(A)$ has at least two points. We then choose 
a neighbourhood $U_{n}$ of $z_{n}$ so that $g\left(U_{n}\right) \subset V_{n}$. Now $g\left(f^{p k_{n}}\left(U_{n}\right)\right)=h^{p k_{n}}\left(g\left(U_{n}\right)\right) \subset$ $h^{p k_{n}}\left(V_{n}\right) \subset \mathbb{C} \backslash A$ so that $f^{p k_{n}}\left(U_{n}\right) \subset \mathbb{C} \backslash B$ for all $p \in \mathbb{N}$. It follows that $z_{n} \in U_{n} \subset$ $F\left(f^{k_{n}}\right)=F(f)$, which gives a contradiction. This completes the proof of (2).

Suppose that $g$ is continuous and that $g(\mathbb{C})$ is an open set, which is true, in particular, if $g$ is an open mapping, and that (1) holds. Now $h^{n}(g(\mathbb{C}))=g\left(f^{n}(\mathbb{C})\right) \subset g(\mathbb{C})$ for all $n \geqslant 1$. If $\mathbb{C} \backslash g(\mathbb{C})$ contains at least two points, then the family $\left\{h^{n}: n \geqslant 1\right\}$ is normal in $g(\mathbb{C})$ so that $g(\mathbb{C}) \subset F(h)$. Then $g(J(f)) \subset g(\mathbb{C}) \subset F(h)$, which contradicts (2). This completes the proof of Theorem 1.

\section{Preliminaries for the proof of Theorem 2}

First we need a result on the growth of composite functions.

Lemma 1. Let $v$ be an entire function satisfying $v(0)=0$ and let $u: \mathbb{C} \rightarrow \mathbb{C}$ be a continuous function satisfying the maximum principle, which means that $|u|$ does not have a local maximum. Let $0<\rho<1$ and define $c(\rho)=(1-\rho)^{2} / 4 \rho$. Then

$$
M(r, u \circ v) \geqslant M(c(\rho) M(\rho r, v), u)
$$

for $r>0$. In particular

$$
M(6 r, u \circ v) \geqslant M(M(r, v), u) .
$$

For entire $u$ and $v$ this result was proved by Pólya [22] with an unspecified constant $c(\rho)$ coming from a theorem of Bohr. Hayman [18] gave a sharp form of Bohr's theorem leading to the value of $c(\rho)$ given above. A fairly elementary proof of Lemma 1 was given by Clunie [11, section 3]. Goldstein [16, p. 122] observed that the proofs mentioned do not require that $u$ is entire but only that $u$ satisfies the maximum principle. The continuity of $u$ was assumed here only to ensure that $|u|$ attains its maximum on compact sets so that $M(r, u)=\max _{|z|=r}|u(z)|$ and $M(r, u \circ v)$ are defined.

For the rest of this section let $f$ be a transcendental entire function. Define

$$
I(f)=\left\{z \in \mathbb{C}:\left|f^{n}(z)\right| \rightarrow \infty \text { as } n \rightarrow \infty\right\} .
$$

Eremenko [13] proved that $I(f) \neq \varnothing$ and deduced from this that $J(f)=\partial I(f)$. His proof that $I(f) \neq \varnothing$ does in fact show that there exists $z_{0} \in I(f)$ such that

$$
\left|f^{n+1}\left(z_{0}\right)\right| \sim M\left(\left|f^{n}\left(z_{0}\right)\right|, f\right)
$$

as $n \rightarrow \infty$. Since $\log M(r, f) / \log r \rightarrow \infty$ for transcendental entire $f$ it follows from (5) that

$$
\frac{\log \left|f^{n+1}\left(z_{0}\right)\right|}{\log \left|f^{n}\left(z_{0}\right)\right|} \rightarrow \infty
$$

as $n \rightarrow \infty$ and this implies that

$$
\frac{\log \log \left|f^{n}\left(z_{0}\right)\right|}{n} \rightarrow \infty
$$

as $n \rightarrow \infty$. In particular, as already mentioned in the introduction,

$$
\frac{\log \log M\left(R, f^{n}\right)}{n} \rightarrow \infty
$$

as $n \rightarrow \infty$ if $R>\left|z_{0}\right|$. 
We shall deduce the following lemma from Eremenko's method.

Lemma 2. For each $R>0$ there exists $z_{0} \in \mathbb{C}$ such that

$$
\left|f^{n}\left(z_{0}\right)\right| \geqslant 2 M\left(R, f^{n}\right)
$$

for all $n \in \mathbb{N}=\{0,1,2, \ldots\}$.

Proof. Since $f$ is transcendental and since $\log M(r, f)$ is convex in $\log r$

$$
\frac{M(2 r, f)}{M(r, f)} \rightarrow \infty
$$

as $r \rightarrow \infty$. We may thus assume without loss of generality that

$$
M(2 r, f) \geqslant 4 M(r, f)
$$

for $r \geqslant R$. By Eremenko's result there exists $z_{0} \in I(f)$ satisfying (5). We may assume that

$$
\left|f^{n+1}\left(z_{0}\right)\right| \geqslant \frac{1}{2} M\left(\left|f^{n}\left(z_{0}\right)\right|, f\right)
$$

for all $n \in \mathbb{N}$ and that

$$
\left|z_{0}\right| \geqslant 2 R
$$

because otherwise we may replace $z_{0}$ by $f^{k}\left(z_{0}\right)$ for a sufficiently large $k$.

We shall prove (7) by induction. Now (10) says that (7) holds for $n=0$ and so we assume that (7) holds for some $n \in \mathbb{N}$. Combining this with (8) and (9) we deduce that

$\left|f^{n+1}\left(z_{0}\right)\right| \geqslant \frac{1}{2} M\left(\left|f^{n}\left(z_{0}\right)\right|, f\right) \geqslant 2 M\left(\frac{\left|f^{n}\left(z_{0}\right)\right|}{2}, f\right) \geqslant 2 M\left(M\left(R, f^{n}\right), f\right) \geqslant 2 M\left(R, f^{n+1}\right)$.

This completes the proof of Lemma 2.

We now fix a value $R>0$ so large that (6) is satisfied and, as noted in the introduction, define

$$
A(f)=\left\{z \in \mathbb{C}: \text { there exists } L \in \mathbb{N} \text { such that }\left|f^{n}(z)\right|>M\left(R, f^{n-L}\right) \text { for } n>L\right\} .
$$

Clearly we have $A(f) \subset I(f)$.

Lemma 3. $J(f) \subset \overline{A(f)}$.

Proof. It follows from the definition of $A(f)$ that $A(f)$ is completely invariant. Hence $\overline{A(f)}$ is completely invariant. Lemma 2 says that $A(f)$ is not empty and in fact is an infinite set. The conclusion now follows from the fact already mentioned in the introduction that the Julia set is the smallest closed completely invariant set with at least three points.

We note here that it also follows as in [13] that $J(f)=\partial A(f)$. We also note that if $R>R_{0}:=\min _{z \in J(f)}|z|$, then (6) is satisfied. Moreover, $A(f)$ does not depend on the choice of $R$ as long as $R>R_{0}$. We shall, however, not need these observations.

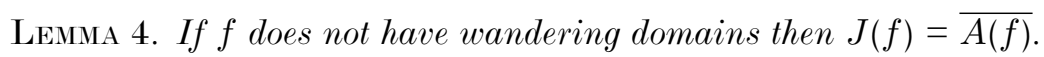

Proof. Because of Lemma 3 and since $J(f)$ is closed it suffices to prove that $A(f) \subset$ $J(f)$. Suppose now that $z_{0} \in A(f) \cap F(f)$. Since $f$ has no wandering domains, there exists $m \in \mathbb{N}$ such that $f^{m}\left(z_{0}\right)$ is in a periodic component of $F(f)$ where the iterates 
of $f$ tend to $\infty$. Such periodic components of $F(f)$ are called Baker domains. Baker ([4, theorem 1], see also [6, lemma 7]) proved that if $w$ is in a Baker domain, then

$$
\log \left|f^{n}(w)\right|=O(n)
$$

as $n \rightarrow \infty$. It follows that

$$
\log \left|f^{n}\left(z_{0}\right)\right|=O(n)
$$

as $n \rightarrow \infty$. On the other hand, (6) and the definition of $A(f)$ imply that

$$
\frac{\log \log \left|f^{n}\left(z_{0}\right)\right|}{n} \rightarrow \infty
$$

as $n \rightarrow \infty$. Clearly (11) and (12) give a contradiction and hence $A(f) \cap F(f)=\varnothing$. It follows that $A(f) \subset J(f)$.

\section{Proof of Theorems 2 and 4}

We will deduce Theorem 2 from the following result.

Theorem 5. Let $f$ and $h$ be transcendental entire functions and let $g: \mathbb{C} \rightarrow \mathbb{C}$ be open and continuous such that $g \circ f=h \circ g$. Then $g^{-1}(A(h)) \subset A(f)$ and $g^{-1}(\overline{A(h)}) \subset \overline{A(f)}$.

Proof. It is well known and not difficult to prove that $f^{2}$, the second iterate of $f$, has a fixed point (see, for example [15]). Moreover, it is easy to see that $A\left(f^{2}\right)=A(f)$. Thus it is no loss of generality to assume that $f$ has a fixed point. We may place this fixed point at the origin so that $f(0)=0$ because otherwise we can conjugate $f, g$ and $h$ by a suitable linear map.

For $R>0$ we shall set $D_{R}=\{z \in \mathbb{C}:|z|<R\}$ and $C_{R}=\{z \in \mathbb{C}:|z|=R\}$. We choose $R$ so large such that $D_{R} \cap J(h) \neq \varnothing$ and such that (6) holds in the form stated and also holds with $f$ replaced by $h$. Thus this value of $R$ can be used in the definition of $A(f)$ and $A(h)$. We now choose $S>0$ such that $g\left(C_{6 R}\right) \subset D_{S}$ and such that $C_{S}$ contains no Fatou exceptional value of $h$. Recall that $a \in \mathbb{C}$ is called a Fatou exceptional value of $h$ if $h^{-1}(a) \subset\{a\}$. Since $h$ has at most one Fatou exceptional value this choice of $S$ is clearly possible. It now follows that there exists $M \in \mathbb{N}$ such that $h^{M}\left(D_{R}\right) \supset C_{S}$. We deduce that if $n \in \mathbb{N}$, then $h^{n+M}\left(D_{R}\right) \supset h^{n}\left(C_{S}\right)$ and hence

$$
M\left(R, h^{n+M}\right) \geqslant M\left(S, h^{n}\right) \geqslant \max _{w \in g\left(C_{6 R}\right)}\left|h^{n}(w)\right|=M\left(6 R, h^{n} \circ g\right)=M\left(6 R, g \circ f^{n}\right) .
$$

Using Lemma 1 we obtain

$$
M\left(R, h^{n+M}\right) \geqslant M\left(M\left(R, f^{n}\right), g\right) .
$$

Suppose now that $z_{0} \in \mathbb{C}$ is such that $g\left(z_{0}\right) \in A(h)$. Then there exists $L \in \mathbb{N}$ such that

$$
\left|g\left(f^{n}\left(z_{0}\right)\right)\right|=\left|h^{n}\left(g\left(z_{0}\right)\right)\right| \geqslant M\left(R, h^{n-L}\right)
$$

for $n>L$. It follows that

$$
M\left(\left|f^{n}\left(z_{0}\right)\right|, g\right) \geqslant M\left(R, h^{n-L}\right) .
$$

Combining (13) and (14) yields

$$
M\left(\left|f^{n}\left(z_{0}\right)\right|, g\right) \geqslant M\left(M\left(R, f^{n-L-M}\right), g\right)
$$


and hence

$$
\left|f^{n}\left(z_{0}\right)\right| \geqslant M\left(R, f^{n-L-M}\right)
$$

for $n>L+M$. It follows that $z_{0} \in A(f)$. We thus have $g^{-1}(A(h)) \subset A(f)$ and since $g$ is open this also implies that $g^{-1}(\overline{A(h)}) \subset \overline{A(f)}$. This completes the proof of Theorem 5.

Proof of Theorem 2. Suppose that $A(f) \subset J(f)$. As already noted in the proof of Lemma 4 this implies that $J(f)=\overline{A(f)}$. Lemma 4 says that this is satisfied in particular if $f$ has no wandering domains. Because of Theorem 1 we only have to prove $(3)$. By Lemma 3 we have $J(h) \subset \overline{A(h)}$. Theorem 5 now yields

$$
g^{-1}(J(h)) \subset g^{-1}(\overline{A(h)}) \subset \overline{A(f)}=J(f) .
$$

Proof of Theorem 4. Suppose that $f$ has no wandering domains but that $h$ has a wandering domain $V$. By the last statement of Theorem $1, g^{-1}(V) \neq \varnothing$. Note that the set $g^{-1}(V)$ is open, and let $U$ be a component of $g^{-1}(V)$. Then $U$ is a component of $F(f)$ by Theorem 2. For $n \in \mathbb{N}$ we have $g\left(f^{n}(U)\right)=h^{n}(g(U)) \subset h^{n}(V)$ and thus $g\left(f^{m}(U)\right) \cap g\left(f^{n}(U)\right)=\varnothing$ for $m \neq n$. It follows that $f^{m}(U) \cap f^{n}(U)=\varnothing$ for $m \neq n$ so that $U$ is a wandering domain of $f$. This is a contradiction.

\section{Proof of Theorem 3}

Suppose the assumptions of Theorem 3 be satisfied. If $g=c$ is constant and satisfies (1) then $c=h(c)$. Since $h$ has only countably many fixed points, there are only countably many such functions $g$. So we may assume from now on that $g$ is non-constant.

Note that there exists $p \in \mathbb{N}$ such that $f^{p}$ has a repelling fixed point; (see [2] for the case that $f$ is transcendental). If (1) holds for some $g$, then $g$ satisfies also (1) with $f, h$ replaced by $f^{p}, h^{p}$. Therefore by replacing $f, h$ by $f^{p}, h^{p}$, if necessary, without changing notation, we may assume that $f$ has a repelling fixed point $\xi$. The assumption that $h$ is not the identity map is no longer needed, since $g$ is nonconstant, so that we do not have to worry about the possibility that some iterate of $h$ might be the identity map.

There is a disk $C=B(\xi, \rho)=\{z:|z-\xi|<\rho\}$ centred at $\xi$ such that there is a univalent branch $F$ of $f^{-1}$ defined in $C$, fixing $\xi$ and mapping $C$ into a relatively compact subset of $C$, such that $F^{n}(z) \rightarrow \xi$ uniformly for $z \in C$ as $n \rightarrow \infty$, and such that $f$ is univalent in $C$.

We write $C_{q}=B(\xi, \rho / q)$ for $q \geqslant 1$. Then each $C_{q}$ has the same properties as $C$, mentioned above. All properties are clear except possibly $\overline{F\left(C_{q}\right)} \subset C_{q}$ for all $q \geqslant 1$. However, even this can be satisfied, for all $q \geqslant 1$, by taking $\rho$ small enough since $\left|F^{\prime}(\xi)\right|=1 /\left|f^{\prime}(\xi)\right|<1$.

Let $\eta$ be a given repelling fixed point of $h$ (we will show later that there is at least one such point $\eta$ provided that there is some non-constant entire $g$ satisfying (1)). There is a disk $K$ centred at $\eta$ such that there is a univalent branch $H$ of $h^{-1}$ defined in $K$, fixing $\eta$ and mapping $K$ into a relatively compact subset of $K$, such that $H^{n}(z) \rightarrow \eta$ uniformly for $z \in K$ as $n \rightarrow \infty$, and such that $h$ is univalent in $K$.

Fix $q \geqslant 1$. Since $\xi \in J(f)$, there are $a \in C_{q} \backslash\{\xi\}$ and $p \geqslant 1$ such that $f^{p}(a)=a$. From now on, let $a$ (and hence $p$ ) be fixed by $q$. Next note that $h^{p}$ has only finitely many fixed points in $K$, and pick such a fixed point $b$. 
We write $P(f, h, \eta, q, b)$ for the set of all non-constant entire functions $g$ that satisfy (1) together with $g(\xi)=\eta, g\left(C_{q}\right) \subset K$, and $g(a)=b$. Note that the choice of $q$ implied the choice of $a$ and $p$.

Suppose that $g, \gamma \in P(f, h, \eta, q, b)$. We seek to prove that $g=\gamma$. This then shows that each set $P(f, h, \eta, q, b)$ has at most one element.

So we assume that $g, \gamma \in P(f, h, \eta, q, b)$. Define $a_{n}=F^{n}(a) \in C_{q}$ and $b_{n}=H^{n}(b) \in$ $K$ for $n \geqslant 0$. Note that $\lim _{n \rightarrow \infty} a_{n}=\xi$.

We have $g\left(a_{n}\right) \in K$. By induction, we show that $g\left(a_{n}\right)=b_{n}$ for all $n \geqslant 0$. This is true for $n=0$. Suppose that it is true for $0 \leqslant n \leqslant k-1$ for some $k \geqslant 1$. The point $z=a_{k}$ is the unique solution in $C_{q}$ to the equation $f(z)=a_{k-1}$, and the point $w=b_{k}$ is the unique solution in $K$ to the equation $h(w)=b_{k-1}$. Now $g\left(a_{k}\right) \in K$ and

$$
h\left(g\left(a_{k}\right)\right)=g\left(f\left(a_{k}\right)\right)=g\left(a_{k-1}\right)=b_{k-1}
$$

so that $g\left(a_{k}\right)=b_{k}$, as required.

The same argument applies with $g$ replaced by $\gamma$, and we deduce that $g\left(a_{n}\right)=\gamma\left(a_{n}\right)$ for all $n \geqslant 1$. Since $g$ and $\gamma$ are entire and $\lim _{n \rightarrow \infty} a_{n}=\xi$ is finite, it follows that $g \equiv \gamma$, as desired.

Note that $h$ has only countably many repelling fixed points and that for each such point $\eta$ as above, once $K$ and $q$ have been chosen (and hence $a$ and $p$ have also been chosen), there are only finitely many fixed points $b$ of $h^{p}$ in $K$. Therefore it now suffices to show that if $g$ is a non-constant entire function satisfying (1) then $g \in P(f, h, \eta, q, b)$ for some repelling fixed point $\eta$ of $h$, some $q \geqslant 1$, and some fixed point $b$ of $h^{p}$ in $K$, where $p$ (as well as $a$ in the condition $g(a)=b$ ) is determined by $q$.

To see this, first note that by (1),

$$
g(\xi)=g(f(\xi))=h(g(\xi))
$$

so that $g(\xi)$ is a fixed point of $h$. Suppose that $f^{\prime}(\xi)=\lambda$ so that $|\lambda|>1$, and that $g(z)=g(\xi)+A_{\mu}(z-\xi)^{\mu}+O\left((z-\xi)^{\mu+1}\right)$ as $z \rightarrow \xi$, where $A_{\mu} \neq 0$ and $\mu \geqslant 1$. Consideration of the power series expansions of both sides of (1) close to $\xi$ shows that then $h^{\prime}(g(\xi))=\lambda^{\mu}$ so that $\left|h^{\prime}(g(\xi))\right|>1$. (Note that this follows immediately by differentiating (1) if $g^{\prime}(\xi) \neq 0$.) Thus $g(\xi)$ is a repelling fixed point of $h$, hence one of the points $\eta$ considered above; this also shows that if such a $g$ exists, then $h$ has at least one repelling fixed point.

If $\eta=g(\xi)$ and a neighbourhood $K$ of $\eta$ is given, then, by continuity, there is $q \geqslant 1$ such that $g\left(\overline{C_{q}}\right) \subset K$.

If $\eta=g(\xi)$ and $K$ and $q \geqslant 1$, and hence $a$ and $p$ also, are given, note that, by (1),

$$
g(a)=g\left(f^{p}(a)\right)=h^{p}(g(a))
$$

and that $g(a) \in K$, so that $b=g(a)$ is a fixed point of $h^{p}$ in $K$, as required. Now $g \in P(f, h, \eta, q, b)$. This completes the proof of Theorem 3 .

Acknowledgements. This research was initiated while A.H. was visiting the Christian Albrechts University of Kiel. He would like to thank the Department of Mathematics for its kind hospitality.

\section{REFERENCES}

[1] I. N. BAKer. Permutable entire functions. Math. Z. 79 (1962), 243-249.

[2] I. N. BaKer. Repulsive fixpoints of entire functions. Math. Z. 104 (1968), 252-256. 
[3] I. N. Baker. Wandering domains in the iteration of entire functions. Proc. London Math. Soc. (3) 49 (1984), 563-576.

[4] I. N. BAKER. Infinite limits in the iteration of entire functions. Ergodic Theory Dynamical Systems 8 (1988), 503-507.

[5] A. F. Beardon. Iteration of rational functions (Springer, 1991).

[6] W. BergweIler. Iteration of meromorphic functions. Bull. Amer. Math. Soc. (N.S.) 29 (1993), $151-188$.

[7] W. Bergweiler. Invariant domains and singularities. Math. Proc. Cambridge Phil. Soc. 117 (1995), 525-532.

[8] W. Bergweiler. On the Julia set of analytic self-maps of the punctured plane. Analysis 15 (1995), 251-256.

[9] W. Bergweiler and Y. F. Wang. On the dynamics of composite entire functions; Ark. Mat. 36 (1998), 31-39.

[10] L. Carleson and T. W. Gamelin. Complex dynamics (Springer, 1993).

[11] J. G. Clunie. The composition of entire and meromorphic functions; in Mathematical essays dedicated to to A. J. Macintyre (ed. H. Shankar) (Ohio Univ. Press, 1970), pp. 75-92.

[12] A. Douady and J. H. Hubbard. On the dynamics of polynomial-like mappings. Ann. Sci. École Norm. Sup. (4) 18 (1985), 287-343.

[13] A. E. Eremenko. On the iteration of entire functions; in Dynamical systems and ergodic theory, Banach Center Publ., vol. 23 (Polish Scientific Publishers, Warsaw, 1989), pp. 339-345.

[14] P. Fatou. Sur l'itération analytique et les substitutions permutables. J. Math. (9) 2 (1923), $343-384$.

[15] P. Fatou. Sur l'itération des fonctions transcendantes entières. Acta Math. 47 (1926), 337-360.

[16] R. Goldstein. On certain compositions of functions of a complex variable. Aequationes Math. 4 (1970), 103-126.

[17] R. Goldstein and F. Gross. Solutions to Problem 5329. Amer. Math. Monthly 73 (1966), 904-905.

[18] W. K. Hayman. Some applications of the transfinite diameter to the theory of functions. $J$. Anal. Math. 1 (1951), 155-179.

[19] G. Julia. Mémoire sur la permutabilité des fractions rationnelles. Ann. Sci. École Norm. Sup. (3) 39 (1922), 131-215.

[20] J. K. Langley. Permutable entire functions and Baker domains; to appear in Math. Proc. Cambridge Phil. Soc.

[21] J. Milnon. Dynamics in one complex variable: introductory lectures. Stony Brook Institute for Mathematical Sciences, Preprint 1990/5.

[22] G. Pólya. On an integral function of an integral function. J. London Math. Soc. 1 (1926), $12-15$

[23] N. Steinmetz. Rational iteration (de Gruyter, 1993)

[24] D. Sullivan. Quasiconformal homeomorphisms and dynamics I: Solution of the Fatou-Julia problem on wandering domains. Ann. Math. 122 (1985), 401-418. 